\title{
Características Socioeconómicas y Salud en Personas Pobres y Desplazadas
}

\author{
Fernando Juárez ${ }^{1}$ \\ Universidad del Rosario \\ Ángela Guerra \\ Universidad Pedagógica y Tecnológica de Colombia
}

\begin{abstract}
RESUMEN - Se estudió la relación entre aspectos socioeconómicos, desplazamiento, eventos traumáticos, salud, trastorno por estrés postraumático (TEPT) y coocurrencia, en 93 personas pobres. Se aplicó la Escala de Trauma Davidson (DTS) y el Cuestionario de Salud General de Goldberg (GHQ-28); 50,5\% de los participantes tuvieron TEPT y 74,2\% tuvo problemas de salud. Existió coocurrencia entre el TEPT y los problemas de salud, siendo la asociación elevada para la ansiedad (GHQ-28) y la hiperreactivación (DTS). En la existencia de problemas de salud, influyó la puntuación en el DTS. En la existencia de TEPT influyó la puntuación en el GHQ-28, el departamento emisor y el tipo de desplazamiento. La puntuación en la DTS y el departamento emisor influyeron en la existencia de coocurrencia.
\end{abstract}

Palabras Clave: Pobreza, desplazamiento forzado, trastorno por estrés postraumático, salud mental, coocurrencia.

\section{Características Sócio-Econômicas e Saúde em Pessoas Pobres e Refugiadas}

\begin{abstract}
RESUMO - Foi estudada a relação entre aspectos socioeconômicos, deslocamento, eventos traumáticos, saúde, transtorno de estresse pós-traumático (TEPT) e co-ocorrência em 93 pessoas de baixa renda. Foi aplicada a Escala de Trauma Davidson (DTS) e o Questionário de Saúde Geral de Goldberg (GHQ-28). 50,5\% dos participantes foram descritos com TEPT e 74,2\% apresentaram problemas de saúde. Existiu co-ocorrência entre o TEPT e os problemas de saúde, sendo a associação elevada para a ansiedade (GHQ-28) e a hiperreatividade (DTS). Na existência de problemas de saúde houve influemcia da pontuação do DTS. Na existência de TEPT houve influencia da pontuação no GHQ-28, o estado de origem e o tipo de deslocamento. A pontuação na DTS e o departamento de origem influenciaram na existência da co-ocorrência.
\end{abstract}

Palavras-Chave: Pobreza, deslocamento forçado, transtorno de estresse pós-traumático, saúde mental, co-ocorrência.

\section{Socioeconomic Characteristics and Health in Poor and Displaced People}

\begin{abstract}
The relationship between social and economic aspects of displacement, traumatic events, health, posttraumatic stress disorder (PTSD) and co-occurrence was studied in a sample of 93 poor and displaced people. The Davidson Trauma Scale (DTS), and Goldberg's General Health Questionnaire (GHQ-28) were applied. Of all the participants, 50.5\% had PTSD and $74.2 \%$ had health problems. Co-occurrence was observed between PTSD symptoms and health problems, with a strong relation between anxiety (GHQ-28) and hyper-reactivation (DTS). Scores on the DTS were related to the existence of health problems; the scores on the GHQ-28, the issuing department and the type of displacement, all of these were related to PTSD. Scores on the DTS and the issuing department influenced the existence of co-occurrence.
\end{abstract}

Keywords: Poverty, forced displacement, posttraumatic stress disorder, mental health, co-occurrence.

Desde los años 1990, se ha incrementado el número de personas desplazadas en su propio país (Contat, 2001) por conflictos armados (Gaviria, et al., 2002). Colombia tiene una de las mayores poblaciones desplazadas del mundo (Amnistía Internacional, 2004), entre 1985 y 2004, más de 3.400 .000 personas dejaron sus hogares, principalmente población rural (Organización Panamericana de la Salud, Oficina Regional de la Organización Mundial de la Salud, \& Programa de Emergencias y Desastres, 2005), aunque muchos desplazados no buscan ayuda (Gonzáles, 2002) y no figuran en los registros (Falla, Chávez, \& Molano, 2005). Esta situación, que dura

1 Endereço para correspondência: Facultad de Administración, Calle 14, No. 4-80 Bogotá, Colombia. E-mail: fernando_juarez2@yahoo.com años (Bacca, Carillo, Páez, \& Martínez, s.f.) provoca rupturas sociofamiliares, destrucción de empleos, de oportunidades educativas y de acceso a necesidades básicas (Deng, 1998), a pesar de que el Estado debería garantizar los derechos de estas personas (Naranjo, 2004).

Las causas más frecuentes del desplazamiento son amenazas, reclutamiento, homicidios, participación directa o indirecta en enfrentamientos armados, torturas, atentados (Rodríguez, Díaz, Niño, Samudio, \& Silva, 2005) y luchas por las zonas rurales (Wallerstein, 2000), así como eventos traumáticos (Alejo, 2005), los cuales generan crisis personales y familiares (Rodríguez, 2006). Además, la experiencia bélica causa sufrimiento mental y físico (Buendía \& Mira, 1993) y afecta la salud de la población (Naranjo, 2004) 
elevando la morbilidad (Gonzáles, 2002), relacionándose el evento traumático con los problemas psicológicos posteriores (Martín \& Ochotorena, 2004). En Colombia, se ha encontrado, en ciertas poblaciones desplazadas, una prevalencia del 27,6\% de desórdenes mentales (Médicos Sin Fronteras, 2006), cifra muy superior a la de la población general $(7,4 \%)$ (Puertas, Ríos, \& del Valle, 2006). Así, el desplazamiento afecta la salud mental (Mogollón, Vázquez, \& García, 2003) generando alteraciones emocionales, incertidumbre, desesperanza, peores condiciones de vida (Camilo, 2000; Falla, Chávez \& Molano, 2005), somatización (Sacipa, 2003) y trastorno por estrés postraumático (TEPT), el cual se asocia a numerosos eventos (Yehuda, 2002) y es incapacitante (Kessler, 2000).

Las situaciones traumáticas consisten en atentados (Martín \& Ochotorena, 2004), ataques militares, abusos, ser rehén, la tortura o el enterarse de un evento traumático, entre otros (Foa, Davidson, \& Frances, 1999), y la abundancia de las mismas en el desplazamiento, aumenta la prevalencia del TEPT (Santacruz \& Ardila, 2002; Palacios \& Heinze, 2002). En Colombia, su prevalencia de vida se ha estimado en $6,8 \%$ para los hombres y $1,9 \%$ para las mujeres, y la anual en $5,9 \%$ para los hombres y $1,2 \%$ para las mujeres (Torres, 1999), en otros estudios, las mujeres presentaron un mayor riesgo de TEPT (Breslau, Davis, \& Andreski, 1995; Hidalgo \& Davidson, 2000).

El TEPT se acompaña de amnesia disociativa (Rhawn \& Research, 1999), evitación, tensión (Galvez, 2005), pensamientos intrusivos, problemas interpersonales, de trabajo, desconfianza, dificultades de sueño (Goodwin, 1987), activación fisiológica, ansiedad, ira, tristeza o vergüenza (Foa, Ehlers, Clark, Tolin, \& Orsillo, 1999), entre otros síntomas. La comorbilidad, coocurrencia, o presencia de más de un trastorno en una persona (Feinstein, 1970), es común en el TEPT (Palacios \& Heinze, 2002), ya que él mismo origina depresión, fobias, somatizaciones y uso de sustancias (Taboada, 1998), y los antecedentes de otro trastorno pueden generar TEPT (Kessler, 2000). La comorbilidad con la depresión ocurre en población desplazada (Prieto, 2002) y ante múltiples eventos dolorosos, sin recibir atención (Keane \& Wolfe, 1990; Flannery, 1998); además, los trastornos de ansiedad suelen preceder al consumo de sustancias (Caraveo \& Colmenares, 2000), asociándose el TEPT con el consumo de drogas y el alcoholismo (Mordechai, 2003). Esto, se observa también en Colombia (Castaño, 1994), donde la desaparición de allegados conlleva sufrimiento, somatizaciones, trastornos alimenticios, dificultades en el trabajo (Sacipa, 2003), duelo y trastornos del estado de ánimo, comórbidos con el TEPT (Alejo, 2005, Rodríguez, 2006).

Los síntomas traumáticos no suelen mejorar si no se tratan los comórbidos (Yehuda, \& Wong, 2001) y la mayoría de los municipios en Colombia no pueden garantizar la atención en salud mental a esta población (Consultoría para los Derechos Humanos y el Desplazamiento, 2006). Esto, hace necesario analizar más los problemas psicológicos (Schell, Marshall, \& Jaycox, 2004) y las características del grupo a intervenir (Mogollón, et al., 2003), lo que constituye el propósito de este estudio en relación con los aspectos socioeconómicos, y los problemas de salud y de coocurrencia entre el TEPT y otras alteraciones.

\section{Método}

\section{Participantes}

Se eligieron, por conveniencia, 93 participantes, hombres y mujeres pobres y desplazados, mayores de 18 años, registrados en el programa de atención de la Agencia Presidencial para la Acción Social y la Cooperación Internacional de la Unidad Territorial de Boyacá (Departamento de Boyacá), en Colombia.

Como se muestra en la Tabla 1, en mayor proporción los participantes eran mujeres $(n=69 ; 74,2 \%)$, con una edad promedio de 37,72 años $(S D=11,198)$; la ocupación de la mayoría era ama de casa $(n=46 ; 49,5 \%)$, el estado civil era casados o en unión libre $(\mathrm{n}=61 ; 65,6 \%)$, tenían tres hijos ( $\mathrm{n}$ $=29 ; 31,2 \%)$ y vivían con cinco personas $(\mathrm{n}=20 ; 21,5 \%)$. El 59,1\% de los participantes $(\mathrm{n}=55)$ había cursado estudios de primaria, solo el $3,2 \%(\mathrm{n}=3)$ había realizado estudios universitarios.

En la Tabla 2, se observa que la mayoría de los participantes provenían del departamento de Boyacá $(\mathrm{n}=22 ; 23,7 \%)$ y en segundo lugar de Arauca $(\mathrm{n}=11 ; 11,8 \%)$. El 94,6\% (n $=88$ ) se desplazó con sus familias, mayoritariamente en el año $2004(n=22 ; 23,7 \%)$. Las causas más frecuentes fueron las amenazas no especificadas $(n=57 ; 61,3 \%)$ y el conflicto armado en general $(\mathrm{n}=17 ; 18,3 \%)$.

\section{Instrumentos}

Cuestionario de Salud General - 28, de Goldberg (Goldberg's General Health Questionnaire - 28, [GHQ-28]) (Goldberg, 1978). El cuestionario posee un coeficiente de confiabilidad alfa de Cronbach de 0,89, una validez de criterio de 0,92 con el cuestionario de depresión de Beck y de 0,85 con el inventario de ansiedad de rasgo-estado de Spielberger (García, 1999) y coeficientes de validez de entre 0,32 y 0,53 , para las subescalas y el total del cuestionario con el Examen del Estado Actual (Retolaza, et al., 1993). Tiene 4 dimensiones (somático, ansiedad e insomnio, disfunción social y depresión), que hacen referencia a síntomas presentes en las últimas semanas. Las respuestas son tipo Likert con método de corrección 0-0-1-1; al sumar los ítems se obtiene un puntaje total indicativo de la severidad de los disturbios mentales (Martín, 2005). Dispone de un punto de corte, donde una puntuación superior a $5 / 6$ es indicativa de caso probable, con una especificidad del $82 \%$ y una sensibilidad del 84,6\% (Alvarez \& Crespo, 1995) o del $80 \%$ y $60 \%$, respectivamente (Retolaza et al., 1993).

Escala de trauma Davidson (Davidson Trauma Scale, DTS; Davidson, 1996). Mediante 17 ítems, esta escala identifica tres grupos de síntomas del TEPT (Davidson, 1996; Bobes et al., 2000), referentes a la intrusión, evitación e hiperreactivación. Utiliza escalas Likert de 5 puntos, para evaluar la frecuencia y la gravedad de los síntomas durante la última semana (Bobes et al. 2000). Arroja una puntuación total (oscila entre 0-136 puntos), así como puntuaciones de frecuencia y gravedad (entre 0 y 68 puntos). Se recomienda utilizar la puntuación de 40, como punto de corte en el total de la escala (Davidson, 1996). Presenta una consistencia 
Tabla 1. Datos socioeconómicos de los participantes

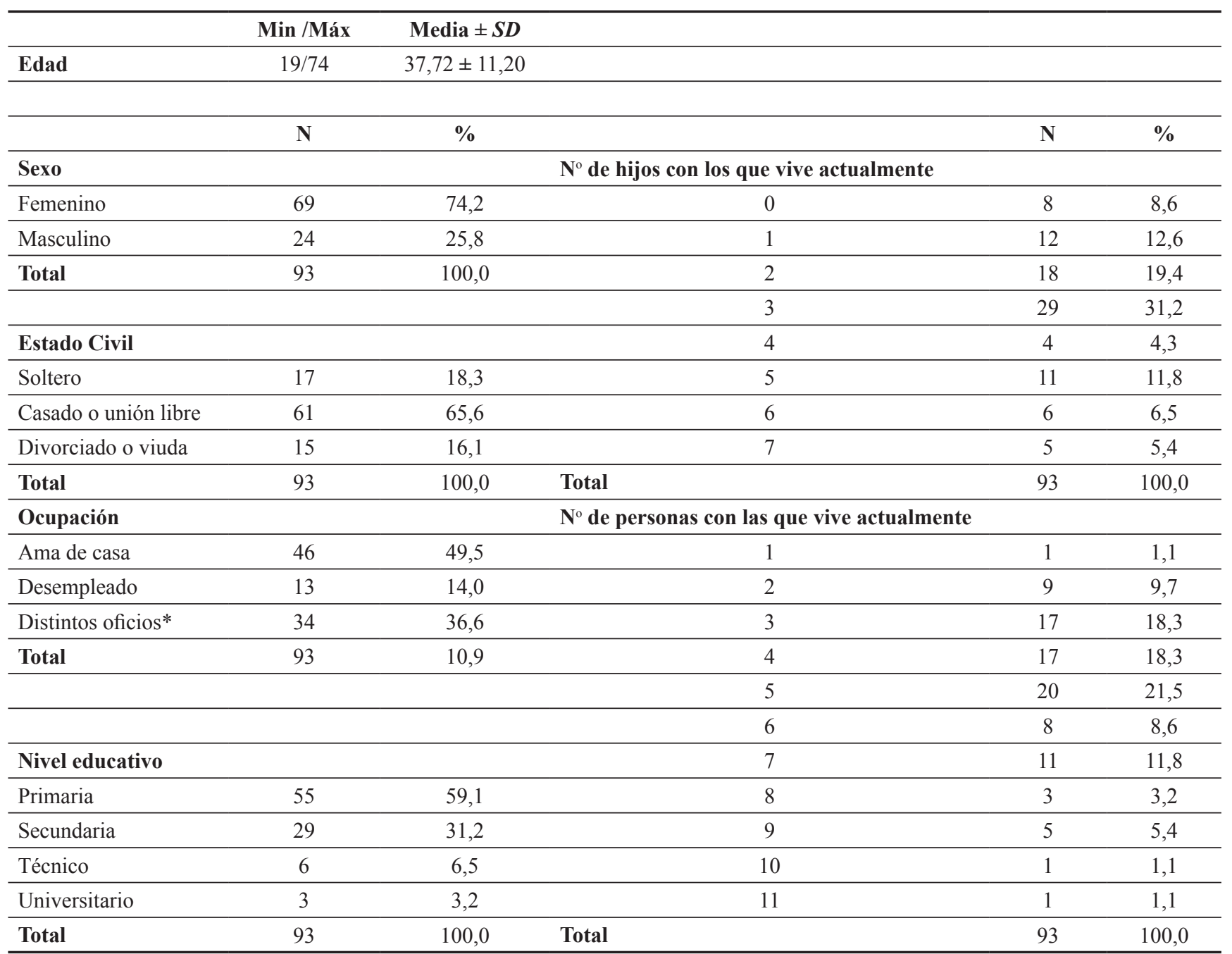

* confeccionista, agropecuario, ladrillero, estilista, jardinero, agricultor, artesana, auxiliar de cocina, cotero, jornalero, electricista, celador.

interna ( $\alpha$ de Crobanch) de 0,99 para el total de la escala, de 0,97 para la frecuencia, y de 0,98 para la gravedad y una fiabilidad test-retest de 0,86 (Bobes et al. 2000). Se ha obtenido un componente de gravedad que explicaba el 24,19 $\%$ de la variancia y otro formado por ítems de intrusión, evitación y embotamiento, que explicaba el $1,34 \%$; en los pacientes con TEPT se han obtenido seis factores, semejantes a los síntomas del trastorno, que explicaban el $26,91 \%$ de la varianza (Bobes, et al. 2000).

\section{Procedimiento}

A medida que los participantes entraban en contacto con el centro de atención y aceptaban voluntariamente formar parte de la investigación, se les aplicaban los instrumentos DTS y GHQ-28. Posteriormente se obtenían los datos socioeconómicos del registro de cada participante en la institución. La investigación fue aprobada por la Agencia Presidencial para la Acción Social y la Cooperación Internacional de la Unidad Territorial de Boyacá.

\section{Resultados}

Tal como se indica en la Tabla 3, el evento traumático más reportado fueron las amenazas $(\mathrm{n}=26 ; 28 \%)$ a él/ella o a la familia, siguiéndole las situaciones generadas por el conflicto armado $(\mathrm{n}=17 ; 18,3 \%) \mathrm{y}$, a continuación, el asesinato de amigos, conocidos o familiares $(\mathrm{n}=15 ; 16,1 \%)$. El 57\% $(\mathrm{n}=53)$ de los participantes refiere haber visto el hecho traumático, mientras que al $36,6 \%(\mathrm{n}=34)$ le ocurrió personalmente; la mayoría de los eventos traumáticos ocurrieron en el año $2004(\mathrm{n}=18 ; 19,4 \%)$.

Como se puede ver en la Tabla 4, en la DTS la media más alta se obtuvo en los síntomas de evitación $(18,87, \mathrm{SD}$ $=14,470)$, obteniéndose un promedio total de 48,23 (SD = $34,716)$. En el GHQ-28, la media más alta se obtuvo en los síntomas de ansiedad e insomnio $(3,42 ; \mathrm{SD}=2,542)$ seguida de los somáticos $(3,39 ; \mathrm{SD}=2,345)$, obteniendo un promedio total de 10,22 $(\mathrm{SD}=6,936)$. Mediante los puntos de corte, se estableció la presencia de TEPT y de problemas mentales. En la tabla, se observa que, en el GHQ-28, el 74,2\% $(\mathrm{n}=69)$ presentaron problemas mentales; en la DTS, el 50,5\% $(\mathrm{n}=$ 47) presentaron TEPT; además, 44 participantes presentaron 
Tabla 2. Datos relativos al desplazamiento

\begin{tabular}{|c|c|c|c|c|c|}
\hline & n & $\%$ & & $\mathbf{n}$ & $\%$ \\
\hline Departamento expulsor & & & Fecha de desplazamiento & & \\
\hline Atlántico, Nariño & $2(1 \mathrm{c} / \mathrm{u})^{*}$ & $2,2(1,1 \mathrm{c} / \mathrm{u})$ & 1990-1994-1999-2007 & $4(1 \mathrm{c} / \mathrm{u})$ & $4,4(1,1 \mathrm{c} / \mathrm{u})$ \\
\hline Bolívar, Caldas, Cesar, Choco, Cundinamarca, Huila & $12(2 \mathrm{c} / \mathrm{u})$ & $12,9(2,2 \mathrm{c} / \mathrm{u})$ & 2000 & 2 & 2,2 \\
\hline Tolima & 3 & 3,2 & 2001 & 7 & 7,5 \\
\hline $\begin{array}{r}\text { Caquetá, Casanare, Antioquia, Norte de Santander, } \\
\text { Santander }\end{array}$ & $25(5 \mathrm{c} / \mathrm{u})$ & $26,9(5,4 \mathrm{c} / \mathrm{u})$ & 2002 & 14 & 15,1 \\
\hline Meta & 8 & 8,6 & 2003 & 14 & 15,1 \\
\hline Guaviare & 10 & 10,8 & 2004 & 22 & 23,7 \\
\hline Arauca & 11 & 11,8 & 2005 & 13 & 14,0 \\
\hline Boyacá & 22 & 23,7 & 2006 & 17 & 18,3 \\
\hline Total & 93 & 100,0 & Total & 93 & 100,0 \\
\hline Causa de desplazamiento & & & Tipo de Desplazamiento & & \\
\hline Amenazas no especificadas & 57 & 61,3 & En solitario & 4 & 4,3 \\
\hline Amenaza de reclutamiento hijo & 6 & 6,5 & Familiar & 88 & 94,6 \\
\hline Amenaza de chantaje & 1 & 1,1 & Con otros & 1 & 1,1 \\
\hline Amenaza de muerte & 1 & 1,1 & Total & 93 & 100,0 \\
\hline Asesinato de la pareja & 2 & 2,2 & & & \\
\hline Asesinato de otros familiares & 2 & 2,2 & & & \\
\hline Asesinato de hijos & 1 & 1,1 & & & \\
\hline Colaborarle al ejercito & 1 & 1,1 & & & \\
\hline Enfrentamientos & 4 & 4,3 & & & \\
\hline Tomas del lugar de residencia & 1 & 1,1 & & & \\
\hline Conflicto armado & 17 & 18,3 & & & \\
\hline Total & 93 & 100,0 & & & \\
\hline
\end{tabular}

*c/u: cada unidad, es decir distribución de la frecuencia total (n) por cada unidad o fecha indicada

coocurrencia de TEPT y otros trastornos mentales, lo que resultó significativo $(\boldsymbol{\varphi}=\mathbf{0 , 4 0 9 ;} p<0,001)$.

En la Tabla 5, se observa la asociación entre los síntomas del TEPT y de otros trastornos mentales, mediante el coeficientes de correlación de Pearson (r); todas las correlaciones resultaron significativas, siendo las más altas las existentes entre la ansiedad $(r=0,769)$ y el total del GHQ-28 $(r=0,780)$, ambos con la hiperreactivación del DTS, y la ansiedad $(r=0,714)$ y el total del GHQ-28 $(r=0,757)$, ambos con el total de la DTS.

Mediante coeficientes Lambda $\left(\lambda_{\mathrm{Y} / \mathrm{X}}\right)$ o ETA, según el tipo de datos, se determinaron las variables socioeconómicas, de desplazamiento y del evento traumático, que se asociaban con la presencia de problemas de salud y TEPT. En los problemas de salud, no resultó significativa ninguna asociación entre las variables anteriores; en el TEPT resultaron significativas el departamento emisor $\left(\lambda_{\mathrm{Y} / \mathrm{X}}=0,435 ; p=0,005\right)$, y el tipo de desplazamiento $\left(\lambda_{\mathrm{Y} / \mathrm{X}}=0,087 ; p=0,041\right)$.

Además, se realizaron ecuaciones de regresión logística, incluyendo las puntuaciones totales de la DTS y del GHQ-28. En la regresión sobre los problemas de salud $\left(\mathrm{R}^{2}=0,403 ; p<0,001\right)$ solamente la puntuación total de la DTS resultó significativa, clasificando cor- rectamente el 74,24\% de los casos (Tabla 6, Modelo 1). En la regresión sobre la presencia de TEPT $\left(\mathrm{R}^{2}=0,494\right.$; $p<0,001)$, resultaron significativas algunas categorías del departamento emisor, del tipo de desplazamiento y el total del GHQ-28, clasificando correctamente el 84,9\% de los casos (Tabla 6, Modelo 2).

De nuevo, mediante coeficientes Lambda $\left(\lambda_{\mathrm{Y} / \mathrm{X}}\right)$ o ETA, se determinaron las variables relevantes en la explicación de la coocurrencia, resultando significativa la ocupación $\left(\lambda_{\mathrm{Y} / \mathrm{X}}=\right.$ $0,159 ; p=0,047)$, el departamento emisor $\left(\lambda_{\mathrm{Y} / \mathrm{X}}=0,432 ; p=\right.$ $0,001)$, la causa del desplazamiento $\left(\lambda_{\mathrm{Y} X}=0,136 ; p=0,03\right)$ y el tipo de trauma $\left(\lambda_{Y / X}=0,250 ; p=0,013\right)$. Se realizaron dos regresiones logísticas sobre la coocurrencia, una con las variables de puntuación total en la DTS y el GHQ-28, y otra en la que también se incluyeron la ocupación, el departamento emisor y la causa del desplazamiento. En la primera $\left(\mathrm{R}^{2}=\right.$ $0,189 ; p<0,001)$, resultó significativa la puntuación total de la DTS, clasificando correctamente el $59,1 \%$ de los casos (Tabla 7, Modelo 1). En la regresión con todas las variables $\left(\mathrm{R}^{2}=0,711 ; p<0,001\right)$ resultó significativa, la puntuación en la DTS y algunas categorías del departamento emisor, clasificando correctamente el $98,96 \%$ de los casos (Tabla 7, Modelo 2). 
Tabla 3. Datos relativos al evento traumático

\begin{tabular}{|c|c|c|c|c|c|}
\hline & $\mathbf{n}$ & $\%$ & & n & $\%$ \\
\hline Evento Traumático & & & $\begin{array}{l}\text { Fecha de evento } \\
\text { traumático }\end{array}$ & & \\
\hline Amenazas (mano armada, pareja, familia, maltrato, reclutamiento) & 26 & 28,0 & $\begin{array}{l}\text { 1986-1994-1998- } \\
2007\end{array}$ & $1 \mathrm{c} / \mathrm{u}^{*}$ & $1,1 \mathrm{c} / \mathrm{u}$ \\
\hline Amenaza de reclutamiento (hijos, esposo) & 5 & 5,4 & 1999 & 5 & 5,4 \\
\hline Asesinato (amigos, conocidos, otros familiares & 15 & 16,1 & 2000 & 2 & 2,2 \\
\hline Asesinato (esposo, hijo) & 6 & 6,1 & 2001 & 8 & 8,6 \\
\hline Cadáver (amigos, conocidos, otros familiares) & 8 & 8,6 & 2002 & 15 & 16,1 \\
\hline Cadáver (esposo, hijo) & 1 & 1,1 & 2003 & 14 & 15,1 \\
\hline $\begin{array}{l}\text { Conflicto armado (enfrentamientos, chantaje, tomas del lugar de } \\
\text { residencia) }\end{array}$ & 17 & 18,3 & 2004 & 18 & 19,4 \\
\hline Disparos, intentos de asesinato & 6 & 6,5 & 2005 & 12 & 12,9 \\
\hline Masacre (amigos, conocidos, familiares) & 5 & 5,4 & 2006 & 15 & 16,1 \\
\hline Reclutamiento (esposo, hijos) & 4 & 4,3 & Total & 93 & 100,0 \\
\hline Total & 93 & 100,0 & & & \\
\hline \multicolumn{6}{|l|}{ Tipo de experimentación } \\
\hline Presenció & 53 & 57,0 & & & \\
\hline Personal & 34 & 36,6 & & & \\
\hline Escuchó & 6 & 6,5 & & & \\
\hline Total & 93 & 100,0 & & & \\
\hline
\end{tabular}

*c/u: cada unidad, es decir distribución de la frecuencia total (n) por cada unidad o fecha indicada

Tabla 4. Datos descriptivos de la DTS y del GHQ-28

\begin{tabular}{|c|c|c|c|c|c|c|c|}
\hline \multicolumn{8}{|c|}{ DTS } \\
\hline Dimensiones & Media & SD & Min. & Máx. & TEPT & n & $\%$ \\
\hline Intrusivos & 13,22 & 11,141 & 0 & 37 & No & 46 & 49,5 \\
\hline Evitación & 18,87 & 14,470 & 0 & 55 & $\mathrm{Si}$ & 47 & 50,5 \\
\hline Hiperreactivación & 16,14 & 12,008 & 0 & 40 & Total & 93 & 100,0 \\
\hline Total & 48,23 & 34,716 & 0 & 128 & & & \\
\hline
\end{tabular}

\section{GHQ-28}

\begin{tabular}{|c|c|c|c|c|c|c|c|}
\hline Dimensiones & Media & SD & Min. & Máx. & Problemas de salud & $\mathbf{n}$ & $\%$ \\
\hline Somáticos & 3,39 & 2,345 & 0 & 7 & No & 24 & 25,8 \\
\hline Ansiedad e Insomnio & 3,42 & 2,542 & 0 & 7 & $\mathrm{Si}$ & 69 & 74,2 \\
\hline Disfunción Social & 1,75 & 1,926 & 0 & 6 & Total & 93 & 100,0 \\
\hline Depresión & 1,66 & 1,959 & 0 & 7 & & & \\
\hline Total & 10,22 & 6,936 & 0 & 25 & & & \\
\hline
\end{tabular}

\section{Coocurrencia de TEPT y problemas de salud}

\begin{tabular}{|c|c|c|c|c|c|}
\hline & & \multicolumn{3}{|c|}{ TEPT (DTS) } & \multirow{2}{*}{$\begin{array}{c}\text { Phi } \\
0,409 * * \\
\end{array}$} \\
\hline & & No & $\mathbf{S i}$ & Total & \\
\hline \multirow[t]{2}{*}{$\begin{array}{l}\text { Problemas de } \\
\text { salud(GHQ-28) }\end{array}$} & No & 21 & 3 & 24 & \\
\hline & $\mathbf{S i}$ & 25 & 44 & 69 & \\
\hline Total & Total & 46 & 47 & 93 & \\
\hline
\end{tabular}

$* * p \leq, 01$ 
Tabla 5. Correlaciones entre las dimensiones y la puntuación total del TEPT con las dimensiones y la puntuación total del Goldberg.

\begin{tabular}{|c|c|c|c|c|c|}
\hline \multirow[t]{2}{*}{ Dimensiones DTS } & \multicolumn{5}{|c|}{$\begin{array}{c}\text { Dimensiones } \\
\text { GHQ-28 }\end{array}$} \\
\hline & Somáticos & Ansiedad & Disfunción Social & Depresión & $\begin{array}{l}\text { Puntuaciones } \\
\text { total GHQ-28 }\end{array}$ \\
\hline Intrusivos & $0,471 * *$ & $0,590 * *$ & $0,439 * *$ & $0,487 * *$ & $0,635^{* *}$ \\
\hline Evitación & $0,494 * *$ & $0,619 * *$ & $0,515^{* *}$ & $0,508 * *$ & $0,681 * *$ \\
\hline Hiperreactivación & $0,577 * *$ & $0,769 * *$ & $0,513 * *$ & $0,568 * *$ & $0,780 * *$ \\
\hline Puntuación total DTS & $0,557 * *$ & $0,714 * *$ & $0,533 * *$ & $0,564 * *$ & $0,757 * *$ \\
\hline
\end{tabular}

$* * p<0,01$

Tabla 6. Coeficientes de la regresión sobre los problemas de salud (Modelo 1) y sobre el TEPT (Modelo 2)

\begin{tabular}{|c|c|c|c|c|}
\hline MODELO 1 & & MODELO 2 & & \\
\hline PROBLEMAS DE SALUD (GHQ-28) & B & TEPT (DTS) & & B \\
\hline \multirow[t]{5}{*}{ Total DTS } & $0,036^{* *}$ & Departamento emisor $^{\mathrm{a}}$ & Bolívar & $-21,936^{*}$ \\
\hline & & & Cesar & $-19,172 *$ \\
\hline & & & Meta & $-3,989 * *$ \\
\hline & & Tipo de desplazamiento & Otro tipo & $15,787 * *$ \\
\hline & & Total GHQ-28 & &, $351 * *$ \\
\hline
\end{tabular}

$* p \leq, 05 ; * * p \leq, 01$, a: Se toma como categoría de referencia el departamento de Antioquia. Se han eliminado las variables no significativas

Tabla 7. Coeficientes de la regresión de los problemas de salud y el TEPT (Modelo 1) y de los problemas de salud, el TEPT y el departamento emisor (Modelo 2) sobre la coocurrencia

\begin{tabular}{|c|c|c|c|c|}
\hline MODELO 1 & & MODELO 2 & & \\
\hline $\begin{array}{c}\text { Solo DTS y } \\
\text { GHQ-28 }\end{array}$ & B & DTS, GHQ-28 y Departamento emisor & & B \\
\hline \multirow[t]{5}{*}{ Total DTS } & $0,028 *$ & Departamento emisor ${ }^{\mathrm{a}}$ & Arauca & $-11,299 *$ \\
\hline & & & Caquetá & $-10,421 *$ \\
\hline & & & Meta & $-10,234 *$ \\
\hline & & & Tolima & $-9,845^{*}$ \\
\hline & & Total DTS & & $0,276^{*}$ \\
\hline
\end{tabular}

$* p \leq, 05,{ }^{\text {a: }}$ Se toma como categoría de referencia el departamento de Antioquia. Se han eliminado las variables no significativas.

\section{Discusión}

En general, las características socioeconómicas de los participantes coinciden con las reportadas en otros estudios similares; la mayoría eran mujeres cabezas de familia (Amnistía Internacional, 2004 y Forero, 2003), con una edad promedio superior a 37 años, por la mortalidad selectiva de hombres entre los 25 y 34 años, y con ausencia de actividad productiva, ya que siendo agricultores, dicha actividad no era viable en sus nuevos contextos, tal como ha sido sugerido por Castillo (2005). El predominio, en nuestro estudio, del estado civil de unión libre, difirió de otro estudio realizado en la localidad de Bogotá, donde fue más frecuente la soltería (Alcaldía Mayor de Bogotá, 2004), aunque ambos coincidieron en el número de hijos ( 2 y 3 ) con los que se vivía. Todos los participantes tuvieron algún nivel educativo, al contrario que en un estudio nacional donde el $11,7 \%$ no tenían ningún tipo de estudios, aunque coincidían en que los participantes mayoritariamente tenían estudios de primaria y secundaria (Castillo, 2005).

El departamento con mayor frecuencia emisora fue Boyacá, seguido por Arauca, lo que no coincide con otros estudios nacionales (Falla et al., 2005); esto se debe a que nuestro trabajo se realizó en Boyacá, recogiendo mayoritariamente la población de ese departamento. El desplazamiento familiar es el tipo de movilización predominante, al igual que en otro estudio ya mencionado (Alcaldía Mayor de Bogotá, 2004). La mayor frecuencia de desplazamiento se produjo en 2004 y 2006, al contrario que en otras investigaciones donde se 
informa que en 2004, hubo una reducción del $41 \%$ respecto a 2003 (Vicepresidencia de la República, s.f.).

En todos los participantes, la principal causa del desplazamiento fueron las amenazas no especificadas, seguidas por el conflicto armado en general, las amenazas de reclutamiento a los hijos y los enfrentamientos armados, lo que coincide también con otros estudios (Bacca et al., s.f., Comité Internacional de la Cruz Roja, s.f. y Organización Panamericana de la Salud \& Programa de Emergencias y Desastres, 2005), donde se encontraron las mismas razones. Además, los principales eventos traumáticos fueron las amenazas, el conflicto armado en general y el asesinato, al igual que en otros trabajos (Médicos Sin Fronteras, 2006; Rodríguez, 2006; Rodríguez et al., 2005). Estos eventos fueron, en su mayoría, presenciados por los participantes, en los años 2002, 2003, 2004 y 2006, lo que coincide con las fechas de desplazamiento más frecuentes, estando de acuerdo con otras explicaciones (Alejo, 2005).

El TEPT se presentó en el 50,5\% de los participantes, lo que indica que no todas las personas desplazadas, sufren este problema (Palacio, s.f.; Buendía y Mira, 1993), sino que su aparición depende de variables personales (Naranjo, 2004; Yeager \& Roberts, 2003), de la interpretación del acontecimiento (Calcedo, 2000), de la vulnerabilidad (Tobal, González, \& López, 2000) o la personalidad premórbida (Alarcón, 2002). Los síntomas de evitación presentaron una mayor frecuencia e intensidad, siguiéndole la hiperreactivación, lo que coincide parcialmente con otro estudio, en el que la reexperimentación obtuvo las puntuaciones más altas, seguida de la hiperreactivación (Palacio, s.f.).

El $74,2 \%$ de los participantes reportó otros cuadros clínicos (GHQ-28), de manera superior a lo obtenido en otros estudios ( $40 \%$ de las personas con sintomatología por violencia de guerra; Mollica et al., 1999), existiendo relación entre la situación traumática y los problemas psicológicos posteriores (Martín \& Ochotorena, 2004). Los síntomas de ansiedad e insomnio presentaron altas puntuaciones, al igual que en otras investigaciones (Mogollón, et al., 2003), habiéndose indicado que cerca del $66 \%$ de estas personas presentan ansiedad (Organización Panamericana de la Salud et al., 2005). En segundo lugar, se situaron los síntomas somáticos, confirmando que el desplazamiento produce respuestas somáticas (Sacipa, 2003). Por su parte, la baja sintomatología de disfunción social coincide con lo obtenido en otro estudio (Rodríguez, 2006) y las puntuaciones en depresión, aunque ratificaron que dichos sentimientos se dan en las personas desplazadas (Mogollón et al., 2003), tuvieron mayor preponderancia en otra investigación (Organización Panamericana de la Salud et al., 2005).

La coocurrencia del TEPT con otros problemas de salud, se presentó en 44, de los 93 participantes, lo que confirma parcialmente, la existencia de la misma (Palacios \& Heinze, 2002). En relación con esto, se presentó una alta correlación de la ansiedad (GHQ-28) con todas las puntuaciones de la DTS, de la hiperreactivación (DTS) con todas las puntuaciones del GHQ-28, y de los totales del DTS y del GHQ-28. Esto no coincide con otras investigaciones, donde se indicó que el TEPT presenta una elevada coocurrencia con trastornos somáticos (Taboada, 1998; Sacipa, 2003) y con cuadros depresivos (Flannery, 1998; Keane \& Wolfe, 1990), en situaciones de guerra y desplazamiento forzoso (Prieto, 2002).

Por otra parte, solamente el departamento de procedencia, el tipo de desplazamiento y las puntuaciones de la GHQ-28 influyeron en la presencia de TEPT, poniendo de manifiesto que los problemas de salud mental son un riesgo para el TEPT (Kessler, 2000). Únicamente las puntuaciones en la DTS, resultaron relevantes, de manera débil, en los problemas de salud, señalando que el TEPT es un riesgo para otros problemas mentales (Taboada, 1998). Así, las variables socioeconómicas, del evento traumático y, en general, del desplazamiento, no tienen influencia en la presencia de problemas mentales en esta población. Esto contrasta con otros estudios, donde se indica que la vivencia directa del evento traumático genera un gran impacto (Rodríguez, 2006), aunque esto se puede explicar por la interpretación que el sujeto hace de la situación (Calcedo, 2000). Debido a la escasa influencia de otras variables, y a la elevada correlación entre el TEPT y los problemas de salud, es posible que exista un bucle de retroalimentación entre ambos problemas.

En cuanto a la coocurrencia, las puntuaciones de la DTS resultaron débilmente significativas cuando se utilizaron junto con la GHQ-28, como predictores de la misma, mientras que se mostraron más relevantes cuando se incluyó también como predictor el departamento emisor; ninguna otra variable resultó relevante. Esto pone de manifiesto que la pertenencia a algún departamento constituye un riesgo para la coocurrencia, siendo el departamento de referencia (Antioquia) el de mayor riesgo.

Finalmente, los problemas encontrados en este estudio se refieren a la homogeneidad de los participantes, lo que si bien permite caracterizar al grupo, impide encontrar asociaciones adecuadas, siendo necesario diversificar la muestra. Otra dificultad fue que la inclusión de variables socioeconómicas y ambientales da lugar a resultados débiles, en ausencia de un modelo de relación entre las mismas.

\section{Referências}

Alarcón, R. (2002). Trastorno por estrés postraumático: Estudios en veteranos de guerra norteamericanos y su relevancia para América Latina. Revista chilena de Neuro-psiquiatría, 40(2), 35-47.

Alcaldía Mayor de Bogotá (2004). El desplazamiento en Bogotá. Una realidad que clama atención. Bogota, Colombia: Departamento Administrativo de Planeación Distrital.

Alejo, E.G. (2005). Aplicación del modelo de estrés postraumático en el estudio del impacto de la violencia sobre la salud mental en población desplazada. MedUNAB, 8(1), 23-28.

Álvarez, E., \& Crespo, M. D. (1995). Morbilidad psiquiátrica en Atención Primaria: resultados de un estudio epidemiológico con el cuestionario GHQ- 28 de Goldberg. Revista de Medicina de la Universidad de Navarra, 40(1), 6-14.

Amnistía Internacional. (2004). Colombia, cuerpos marcados, crímenes silenciados. Violencia sexual contra las mujeres en el marco del conflicto armado. Madrid, España: Amnistía Internacional. 
Bacca, L. J., Carillo, W. R., Páez, J. F., \& Martínez, A. J. (s.f.). La política pública sobre desplazamiento forzado en Colombia: ¿sólo buenas intenciones?.Economía Colombiana, 307, 104113.

Bobes, G. J., Calcedo-Barba, A., García, M., François, M., RicoVillademoros, F., González, M. P., et al. (2000). Evaluación de las propiedades psicométricas de la versión española de cinco cuestionarios para la evaluación del trastorno de estrés postraumático. Acta Española de Psiquiatría, 28(4), 207-218.

Breslau, N.; Davis, G. C., \& Andreski, M. A. (1995). Risk factors for PTSD-related traumatic events: A prospective analysis. Journal of Psychiatry, 152, 529-35.

Buendía, J., \& Mira, J. M. (1993). Estrés y Psicopatología. Madrid, España: Pirámide.

Calcedo, A. (2000). Trastorno de estrés postraumático. Barcelona, España: Masson S. A.

Camilo, G. (2000). Impacto psicológico del desplazamiento forzoso: Estrategias de intervención. In M. N. Bello, E. Martín, \& F. Arias (Eds.), Efectos psicosociales y culturales del desplazamiento. Bogotá, Colombia: Universidad Nacional

Caraveo, J. J., \& Colmenares, E. (2000). Los trastorno psiquiátricos y el abuso de sustancias en México: Panorama epidemiológico. Salud mental, 25(2), 9-15.

Castaño, B. L. (1994). Violencia socio-politica en Colombia: Repercusión en la salud mental de las víctimas. Bogotá, Colombia: Corporación AVRE.

Castillo, O. L. (2005). Poblaciones en situación de desplazamiento forzado en Colombia. Una revisión de las cifras del sistema de información RUT. Cuadernos de Desarrollo Rural, 55, 29-50.

Comité Internacional de la Cruz Roja (s.f.). Colombia. Informe 2004. Retrieved from http://www.icrc.org/Web/spa/sitespa0. nsf/htmlall/colombia-report-050505/\$File/informe_colombia 2004.pdf

Consultoría para los derechos humanos y el desplazamiento (2006). Guía para la aplicación de los Principios Rectores de los Desplazamientos Internos en situaciones de desplazamiento urbano: El caso de Bogotá. Washington D.C., USA: Autor.

Contat, M. (2001). La protección de los desplazados internos afectados por conflictos armados: Concepto y desafíos. Revista Internacional de la Cruz Roja, 843. Retrieved from http://www. icrc.org/web/spa/sitespa0.nsf/html/5TDQ4P

Davidson, J. R. T. (1996). Davidson Trauma Scale. Toronto, Canada: Multi Health Systems Inc.

Deng, F. (1998). Principios rectores de los desplazados internos (DOC.E/CN.4/ 1998/53/ADD.2). Ginebra, Suiza: Naciones Unidas.

Falla, U., Chávez, Y. A., \& Molano, G. (2005). Desplazamiento forzado en Colombia. Análisis documental e informe de investigación en la Unidad de Atención Integral al Desplazado (UAID)-Bogotá. Tabula Rasa, 1, 221-236.

Feinstein, A. R. (1970). The pre-therapeutic classification of comorbidity in chronic disease. Journal of Chronic Disease 23, 455-468.

Flannery, R. (1998). Posttraumatic stress disorder: The victims guide to healing and recovery. New York, USA: Crossroad.

Foa, E. B., Davidson, J. R. T., \& Frances, A. (1999). The expert consensus guideline series: Treatment of post-traumatic stress disorder. Journal of Clinical Psychiatry, 60, 4-76.
Foa, E. B., Ehlers, A., Clark, D., Tolin, D., \& Orsillo, S. (1999). The Post-Traumatic Cognitions Inventory (PTCI): Development and validation. Psychological Assessment. 11, 303-314.

Forero, E. (2003). El desplazamiento interno forzado en Colombia. Washington, D.C., USA: Kellog Institute. The Woodrow Wilson International Center for Scholars \& Fundación Ideas para la Paz.

Gálvez, J. F. (2005). Trastornos por estrés y sus repercusiones neuropsicoendocrinológicas. Revista Colombiana de Psiquiatría, 34(1), 77-100.

García, C. R. V. (1999). Manual para la utilización del cuestionario de salud general de Goldberg. Adaptación cubana. Revista Cubana de Medicina General Integral, 15(1), 88-97.

Gaviria, M.; Echeverri, E.; Goméz, J.; Peñaranda, F.; Olaya, A.; \& Yepes, C. (2002). Análisis de la situación de salud de la población desplazad y no desplazada en Medellín. Retrieved from http//www.disasterinfo/desplazados/informes/OPS/ perdilmedellin

Goldberg, D. (1978). Manual del General Health Questionnaire. Windsor, England: NFER Publishing.

Gonzáles, M. (2002). Desterrados: El desplazamiento forzado sigue aumentando en Colombia. Convergencia, 9(27), 41-78.

Goodwin, J. (1987). The etiology of combat-related post-traumatic stress disorders. In T. Williams (Ed.), Post-Traumatic Stress Disorders: A handbook for clinicians. Cincinatti, USA: Disabled American Veterans National Headquarters.

Hidalgo, R., \& Davidson J. R. T. (2000). Posttraumatic stress disorder: Epidemiology and health related considerations. Journal of Clinical Psychiatry, 6(7), 5-13.

Keane, T. M., \& Wolfe, J. (1990). Comorbidity in posttraumatic stress disorder. An analysis of community and clinical studies. Journal of Applied Social Psychology, 20, 1776-1788.

Kessler, R. C. (2000). Posttraumatic stress disorder: The burden to the individual and to society. Journal of Clinical Psychiatry, 61(5), 4-12.

Martín, C. (2005). Prevalencia y atención a los trastornos mentales en una comunidad rural. Tesis Doctoral, Universidad de Granada, Granada.

Martín, J. L., \& Ochotorena, J. P. (2004). Trastorno por estrés postraumático en víctimas de situaciones traumáticas. Psicothema, 16(1), 45-49.

Médicos Sin Fronteras (2006). Vivir con Miedo. El ciclo de la violencia en Colombia. Amsterdam, The Netherlands: Oficina de Asuntos Humanitarios MSF.

Mogollón, A. S., Vázquez, M. L., \& García, M. M. (2003). Necesidades en salud de la población desplazada por conflicto armado en Bogotá. Revista Española de Salud Pública, 77(2), 257-266.

Mollica, R., Melnnes, J., Sarajlic, N., Lavelle, J., Sarajlic, I., \& Massagli, M. (1999). Disability associated with psychiatric comorbidity and health status in Bosnian refugees living in Croatia. The Journal of the American Medical Association, 282(5), 433-439.

Mordechai, B. (2003). Lo disruptivo, amenazas individuales y colectivas: El psiquismo ante guerras, terrorismos y catástrofes sociales. Buenos aires, Argentina: Biblos.

Naranjo, G. (2004). Ciudadanía y desplazamiento forzado en Colombia: una relación conflictiva interpretada desde la teoría del reconocimiento. Estudios Políticos (25), 137-160. 
Organización Panamericana de la Salud, Oficina Regional de la Organización Mundial de la Salud, \& Programa de Emergencias y Desastres (2005). Serie Salud y Desplazamiento en Colombia, Comparación de la situación de la salud, entre población en situación de desplazamiento y receptora, en seis ciudades. 2002-2003. Medellín, Colombia: Universidad de Antioquia.

Palacio, J. (s.f.). Estrés postraumático y resistencia psicológica en jóvenes desplazados. Retrieved from http://www.uninorte.edu. co/divisiones/humanidades/apsiun/articulo3. htm

Palacios, L., \& Heinze, G. (2002). Trastorno por estrés postraumático, una revisión del tema (Primera parte). Salud Mental, 25(3), 19-26.

Prieto, A. (2002). Salud Mental: Situación y Tendencias. Retrieved from http://www. revmed.unal.edu.co/revistasp/v4n1/v4n1 ta1. htm

Puertas, G, Ríos, C., \& del Valle, H. (2006). Prevalencia de trastornos mentales comunes en barrios marginales urbanos con población desplazada en Colombia. Revista Panamericana de Salud Publica, 20(5), 324-30.

Retolaza, A., Mostajo, A., de la Rica, J. R., Oiaz, A., Perez, J., Aramberri, I., \& Marquez, I. (1993). Validación del cuestionario de salud general de Goldberg (versión 28 ítems) en consultas de atención primaria. Revista de la Asociación Española de Neuropsiquiatría, (13)46, 187-194.

Rhawn, J., \& Research, B. (1999). The neurology of traumatic "dissociative" amnesia. Commentary and literature review. Child Abuse \& Neglect, 23, 715-727.

Rodríguez, M. C. (2006). Eficacia de un programa de grupo estructurado en estrategias de afrontamiento para DSPT en adultos y adolescentes en situación de desplazamiento. Universitas Psychologica, 5(2), 259-274.

Rodríguez, M. C., Díaz, P., Niño, S., Samudio, M., \& Silva, M. (2005). El desplazamiento como generador de crisis: Un estudio en adultos y adolescentes. Terapia Psicológica, 23, 33-43.

Sacipa, S. (2003). Lectura de los significados en historias del desplazamiento y una organización comunitaria por la paz. Universitas Psychologica, 2(1), 49-65.
Santacruz, H., \& Ardila, S. (2002). Fundamentos de psiquiatría clínica: Niños, adolescentes y adultos. ( $1^{\text {a }}$ Ed.). Bogotá, Colombia: CEJA.

Schell, T. L., Marshall, G. N., \& Jaycox, L. H. (2004). All symptoms are not created equal: The prominent role of hyperarousal in the natural course posttraumatic psychological distress. Journal of Abnormal Psychology, 113, 189-197.

Taboada, M. L. (1998). Eventos traumáticos y reacciones de estrés: Identificación y manejo en una situación de desastre natural. Revista Electrónica de Psiquiatría, 2(4). Retrieved from http:// www.psiquiatria.com/psiquiatria(vol2mum4/art_7.htm

Tobal, J. J. M., González, H., \& López, E. (2000). Estrés postraumático: Hacia una integración de aspectos psicológicos y neurobiológicos. Ansiedad y Estrés, 6(2-3), 255-280.

Torres, G. Y. (1999). Epidemiología de los trastornos psiquiátricos - Estudio Nacional Colombia. Medellín, Colombia.: Facultad de Medicina CES.

Vicepresidencia de la República (s.f.). Desplazamiento forzado. Retrieved from http://www.derechoshumanos.gov.co/ observatorio/indicadores/2006/desplazamiento.pdf

Wallerstein, N. (2000). Violencia en Colombia: Reflexiones de una profesora visitante. Salud publica, 18(2), 101-115.

Yeager, K. R., \& Roberts, A. R. (2003). Differentiating among stress, acute stress disorder, crisis episodes, trauma, and PTSD: Paradigm and treatment goals. Brief Treatment and Crisis Intervention 3, 3-25.

Yehuda, R. (2002). Posttraumatic stress disorder. Current concepts. The New England Journal of Medicine, 346(2), 108-114.

Yehuda, R., \& Wong, Ch. (2001). Etiology and biology of posttraumatic stress disorder: Implications for treatment. Psychiatric Clinics of North America, 8, 109-134.

Recebido em 10.05.2010

Primeira decisão editorial em 24.08.2010

Versão final em 08.09.2010

Aceito em 26.10.2010 